\title{
Atuação do enfermeiro nos cuidados em Unidades de Terapia Intensiva Neonatal: Revisão integrativa da literatura
}

\author{
Nurse's performance in care in Neonatal Intensive Care Units: Integrative literature review \\ Desempeño del enfermero en la atención en Unidades de Cuidados Intensivos Neonatales: Revisión \\ integradora de la literatura
}

Recebido: 02/04/2021 | Revisado: 11/04/2021 | Aceito: 06/05/2021 | Publicado: 19/05/2021

Letícia Erica Neves dos Prazeres

ORCID: https://orcid.org/0000-0002-8155-2960 Centro Universitário Metropolitano da Amazônia, Brasil E-mail: letneves@outlook.com

Maria de Nazare Gonçalves Pereira Ferreira

ORCID: https://orcid.org/0000-0003-0784-4866

Centro Universitário Metropolitano da Amazônia, Brasil

E-mail: nazarepereira480@yahoo.com.br

Marcio Alves Ribeiro

ORCID: https://orcid.org/0000-0002-0803-506X

Centro Universitário Metropolitano da Amazônia, Brasil

E-mail: marcioalves22@hotmail.com

Brenda Tanielle Dutra Barros

ORCID: https://orcid.org/0000-0002-3184-050X

Centro Universitário Metropolitano da Amazônia, Brasil

E-mail: brendatanielle.enf@gmail.com

Rômulo Leno Miranda Barros

ORCID: https://orcid.org/0000-0003-1802-4521

Universidade da Amazônia, Brasil

E-mail: romuloleno@hotmail.com

Catarina Santos Ramos

ORCID: https://orcid.org/0000-0002-2376-8333

Centro Universitário Metropolitano da Amazônia, Brasil

E-mail: catramosblue@gmail.com

Tatiana Fabíola da Silva Lima

ORCID: https://orcid.org/0000-0001-5966-9727

Centro Universitário Metropolitano da Amazônia, Brasil

E-mail: tatiana.fs.lima2@gmail.com

Virgínia Mercês Lara Pessoa Oliveira

ORCID: https://orcid.org/0000-0002-4417-637X

Centro Universitário Metropolitano da Amazônia, Brasil

E-mail: virginiaoliveira@famaz.edu.br

Joyane Mesquita Gois Andrade

ORCID: https://orcid.org/0000-0001-5915-5734

Universidade do Estado do Pará, Brasil E-mail: joymcg17@gmail.com

José Eduardo Resende Campos

ORCID: https://orcid.org/0000-0002-4070-8543

Centro Universitário Metropolitano da Amazônia, Brasil

E-mail: educamposjrc@gmail.com

Aline Costa Martins

ORCID: https://orcid.org/0000-0002-8022-3444 Faculdade Pan Amazônica, Brasil

E-mail: alinemartinsenfa1301@gmail.com

Katielem Melo Vale

ORCID: https://orcid.org/0000-0003-4868-1223

Centro Universitário Metropolitano da Amazônia, Brasil

E-mail: katielem.enf@gmail.com

Marildete da Conceição Paula

ORCID: https://orcid.org/0000-0001-9092-4693

Centro Universitário Metropolitano da Amazônia, Brasil

E-mail: marypaula72@hotmail.com

Lidiane do Socorro Carvalho dos Santos

ORCID: https://orcid.org/0000-0002-6549-5986

Centro Universitário Metropolitano da Amazônia, Brasil

E-mail: lidianecarvalho90@yahoo.com.br 


\title{
Resumo
}

A Unidade de Terapia Intensiva Neonatal (UTIN) está destinada ao atendimento de neonatos com até 28 dias de vida que precisem de um suporte assistencial de alta complexidade em tempo integral. Este estudo teve como objetivo descrever as evidências científicas encontradas na literatura científica do período de 2015 a 2019, sobre a atuação do enfermeiro nos cuidados em unidades de terapia intensiva neonatal. Trata-se de um estudo de abordagem qualitativo, do tipo revisão integrativa da literatura que partiu da seguinte questão norteadora: quais as evidências científicas na literatura acerca da atuação do enfermeiro nos cuidados em unidades de terapia intensiva neonatal, no período de 2015 a 2019? As bases de dados pesquisadas foram BDENF, LILACS e SCIELO. A amostra do estudo foi constituída de onze artigos originais. Para extração dos dados foi utilizado o formulário de Ursi e para discussão, a técnica da Análise de Conteúdo de Bardin. Os resultados foram apresentados e discutidos a partir de duas categorias temáticas: "O papel do enfermeiro na assistência da UTIN" e "A sistematização da assistência de enfermagem (SAE) aplicada em UTIN". Concluiu-se que o uso da Sistematização da Assistência de Enfermagem (SAE) na UTIN faz de grande importância, pois a assistência qualificada não deve se limitar a garantir a sobrevida do prematuro, mas também planejar ações e implementá-las de acordo com o que cuidado irá necessitar.

Palavras-chave: Cuidados de enfermagem; Enfermagem neonatal; UTI neonatal; Diagnóstico de enfermagem.

\begin{abstract}
The Neonatal Intensive Care Unit (NICU) is designed to care for neonates up to 28 days old who need full-time assistance of high complexity. This study aimed to describe the scientific evidence found in the scientific literature from 2015 to 2019, on the role of nurses in care in neonatal intensive care units. This is a qualitative study, an integrative literature review type that started from the following guiding question: what is the scientific evidence in the literature about the role of nurses in care in neonatal intensive care units, in the period from 2015 to 2019? The databases searched were BDENF, LILACS and SCIELO. The study sample consisted of eleven original articles. For data extraction, Ursi's form was used and for discussion, the Bardin Content Analysis technique. The results were presented and discussed from two thematic categories: "The role of nurses in NICU care" and "The systematization of nursing care (SAE) applied in NICU". It was concluded that the use of Systematization of Nursing Assistance (SAE) in the NICU is of fundamental importance, since qualified assistance should not be limited to guarantee the survival of the premature, but also plan actions and implement them according to the needs of Care.
\end{abstract}

Keywords: Nursing care; Neonatal nursing; Neonatal ICU; Nursing diagnosis.

\section{Resumen}

La Unidad de Cuidados Intensivos Neonatales (UCIN) está destinada a la atención de neonatos de hasta 28 días de edad que necesitan asistencia de alta complejidad en tiempo completo. Este estudio tuvo como objetivo describir la evidencia científica encontrada en la literatura científica de 2015 a 2019, sobre el papel del enfermero en la atención en las unidades de cuidados intensivos neonatales. Se trata de un estudio con abordaje cualitativo, tipo revisión integradora de la literatura que partió de la siguiente pregunta orientadora: ¿cuáles son las evidencias científicas en la literatura sobre el rol del enfermero en la atención en las unidades de cuidados intensivos neonatales, en el período 2015-2019? Las bases de datos buscadas fueron BDENF, LILACS y SCIELO. La muestra de estudio estuvo formada por once artículos originales. Para la extracción de datos se utilizó el formulario Ursi y para la discusión la técnica Bardin Content Analysis. Los resultados fueron presentados y discutidos en base a dos categorías temáticas: "El rol del enfermero en el cuidado de la UCIN" y "La sistematización del cuidado de enfermería (SAE) aplicado en la UCIN". Se concluyó que el uso de la Sistematización de la Atención de Enfermería (SAE) en la UCIN es de gran importancia, ya que la atención calificada no debe limitarse a garantizar la supervivencia del prematuro, sino también planificar acciones e implementarlas de acuerdo con los cuidados que necesitará.

Palabras clave: Cuidado de enfermera; Enfermería neonatal; UCI neonatal; Diagnóstico de enfermería.

\section{Introdução}

As Unidades de Terapia Intensiva (UTI) são unidades complexas, as quais se destinam à assistência de pacientes graves, que necessitam de espaço físico específico, recursos humanos especializados e instrumentos tecnológicos avançados, sendo, portanto, unidades de alto custo. Esses espaços se constituem como setores críticos do hospital, os quais são destinados aos pacientes graves que demandam vigilância continua e suporte terapêutico especializado (Ferreira, Amaral \& Lopes, 2017). 
A UTIN está destinada ao atendimento de recém-nascidos (RN) com até 28 dias de vida que precisem de um suporte assistencial diferenciado, de alta complexidade o todo tempo. Com apoio de suporte vital completo, monitoração, equipamentos de reanimação, entre diversos outros, além de serviços auxiliares de apoio (meios diagnósticos e especialidades médicas). Assim, ao setor detém condições essenciais e vitais para a sobrevivência e desenvolvimento do RN no ambiente extra uterino (Coelho et al., 2018).

Nos últimos anos, muitas mudanças têm ocorrido no contexto da assistência hospitalar no Brasil, em especial nas UTI. Uma das principais mudanças diz respeito à evolução tecnológica nessas unidades, o que tem influenciado na mudança do perfil dos pacientes internados nas terapias intensivas, principalmente no que se refere a estadia e nível de atenção requerido, levando em consideração que com mais recursos terapêuticos e tecnológicos à disposição, os casos abordados nesse setor passaram a ser somente os mais graves e mais complexos (Alencar et al., 2017).

Outra importante mudança relaciona-se à humanização da assistência prestada em âmbito hospitalar. Humanizar em UTI significa cuidar do paciente de modo holístico, englobando seus vários aspectos, dentre eles o biológico, emocional, familiar e social. Mas para que isso ocorra de forma integral, é necessária uma qualificação adequada, bem como a tanmbém adequada quantificação dos profissionais que tem atividade na área, para atender às demandas específicas dos pacientes (Ribeiro \& Jatobá, 2015).

A humanização na UTIN se caracteriza com uma das essensiais estratégias para ajudar no desenvolvimento do RN, uma vez que visa diminuir os estressores que causam impacto no desenvolvimento de melhoria e os trabalhadores que compõe equipe de enfermagem são grandes responsáveis por propiciar um ambiente agradável e adequado (Coelho et al., 2018; Silva \& Tonon, 2020).

O desempenho da enfermagem na UTIN reúne diversas atividades, dentre as quais se destacam: procedimentos invasivos especializados, cuidado voltado para o conforto, preservação do repouso do RN, articulação dos processos de cuidado humanos e tecnológicos, entre outros. Tais cuidados irão resultar na recuperação, desenvolvimento e proporcionar a adaptação do RN ao ambiente extra-uterino (Gomes et al., 2019).

À vista disso, segundo Oliveira et al. (2017) a UTIN e o cuidado de enfermagem deve estar focado às necessidades do binômio mãe-filho e os demais familiares, criando uma proposta para o cuidado centrado na família, encorajando-os ao envolvimento afetivo. Tal proposta de enfermagem possui o objetivo de minimizar o tempo de internação, aumentar relações afetivas e o vínculo de confiança entre a família e a equipe profissional (Oliveira et al., 2017).

Ao pessoal da enfermagem, falar sobre o desempenho de suas atividades no contexto da UTIN é interessante, pois o enfermeiro atua integralmente nas etapas da vida, incluindo seu início e nas diversas dimensões do cuidar. Ademais, sabe-se que quando se trata dos cuidados ao grupo neonatal especificamente, as técnicas de cuidados e manobras necessitam serem delicadas, cuidadosas e todas com respaldo científico. E como o conhecimento se atualiza rapidamente, o presente estudo visa proporcionar a este público uma atualização e revisão sobre a temática.

Assim, considera-se este estudo relevante a todos que tiverem acesso ao seu conteúdo, ao aprofundar informações sobre o tema; além da sua importância na formação acadêmica em enfermagem, visto que franqueia o aprendizado sobre as condutas realizadas pelo enfermeiro na assistência em UTIN, destacando a proficiência, a segurança e a humanização do cuidado ao RN.

O estudo objetivou analisar as evidências científicas na literatura acerca da atuação do enfermeiro nos cuidados em unidades de terapia intensiva neonatal. A partir de evidências científicas em periódicos nacionais, publicados no período entre 2015 a 2019. E especificamente levantar os principais diagnósticos de enfermagem aplicados em unidades de terapia intensiva neonatal. 


\section{Metodologia}

Trata-se de um estudo do tipo Revisão Integrativa da Literatura (RIL), de cunho descritivo e versando sobre o modelo qualitativo. A revisão integrativa é um método específico, que agrupa estudos de literatura desenvolvidos independentemente da sua metodologia, para fornecer uma compreensão mais abrangente de um fenômeno particular. Esse método de pesquisa objetiva traçar uma análise sobre o conhecimento já construído em pesquisas anteriores sobre um determinado tema permitindo a geração de novos conhecimentos, pautados nos resultados apresentados pelas pesquisas anteriores de forma sistematizada e rigorosa (Soares et al., 2014; Pereira et al., 2018).

A coleta das informações foram executadas no período de agosto a novembro de 2020, nas bases de dados Bases de dados em enfermagem (BDENF), Literatura Latino-americana e do Caribe em Saúde (LILACS), Scientific Electronic Library Online (SciELO)

Para a construção desta pesquisa foram seguidas as seis fases do processo de elaboração de Revisão Integrativa de Literatura (Teixeira et al., 2014): $1^{\circ}$ etapa - identificação do tema e seleção da hipótese ou questão de pesquisa para a elaboração da revisão integrativa; $2^{\circ}$ etapa - estabelecimento de critérios para inclusão e exclusão de estudos/amostragem ou busca na literatura; $3^{\circ}$ etapa - definição das informações a serem extraídas dos estudos selecionados/categorização dos estudos; $4^{\circ}$ etapa - avaliação crítica dos estudos incluídos na revisão integrativa; $5^{\circ}$ etapa - interpretação dos resultados; $6^{\circ}$ etapa apresentação da revisão/síntese do conhecimento.

Nesse sentido, na $1^{\mathrm{a}}$ etapa foi definida a seguinte pergunta norteadora: "Quais as evidências científicas na literatura acerca da atuação do enfermeiro nos cuidados em unidades de terapia intensiva neonatal, no período de 2015 a 2019 ?”. Esta questão seguiu a lógica da estratégia PICO adaptada, seguindo a recomendação para estudos qualitativos por Joanna Briggs Institutte: P corresponde aos participantes, I ao fenômeno de interesse e Co ao contexto do estudo (Mombaque \& Secoli, 2018).

Desta forma, o "P", relaciona-se aos enfermeiros, o "I", relaciona-se a atuação do enfermeiro na UTIN; o "CO", cuidados em unidades de terapia intensiva neonatal, evidenciado na literatura nos últimos cinco anos.

$\mathrm{Na}$ segunda fase foi realizada a busca nas bases de evidências: Base de Dados de Enfermagem (BDEnf), Literatura Latino-Americano e do Caribe em Ciências da Saúde (LILACS), Scientific Electronic Library Online (SciELO); referentes ao período de 2015 a 2019, estudos nacionais, por meio dos descritores: Cuidado de enfermagem; Enfermagem neonatal; UTI neonatal; Diagnóstico de enfermagem. Foi utilizado o cruzamento dos descritores com utilização dos operadores booleanos "And" e "Or".

Como critérios de inclusão foram definidos: artigos originais completos referentes ao período de 2015 a 2019 , na língua portuguesa e com abordagem ao tema central do estudo. Foram excluídos textos incompletos, resumos, publicações em língua estrangeira, aquelas fora do período definido, sem afinidade com o tema, monografias, trabalhos de conclusão de curso e artigos de revisão teórica.

$\mathrm{Na}$ terceira fase, da extração de dados foi utilizado o formulário padronizado de Ursi (2005) para extração de informações relevantes para compor o estudo. Na quarta fase foi realizada a análise crítica dos estudos aplicando o sistema de classificação de evidências, adotando-se a hierarquia das evidências, em relação ao delineamento de pesquisa: Nível 1: Evidências resultantes da meta-análise; Nível 2: Evidências com delineamento experimental; Nível 3: Evidências de estudo quase-experimental; Nível 4: Evidências de estudo não-experimental ou com abordagem qualitativa; Nível 5: Evidências de relatos de casos ou de experiência; Nível 6: Evidências com opiniões de especialistas. Neste estudo a principal evidencia foi de nível 4, com de abordagem qualitativa (Soares et al., 2014). 
Já na quinta fase, apresentam-se os resultados da pesquisa, onde as principais evidências foram sintetizadas em dois quadros sínteses para encontrar as relações entre os artigos pesquisados, encontrando respostas para a nossa questão de pesquisa e objetivos propostos, após foi comparado ao referencial teórico que fundamentou o estudo.

E na sexta fase foi elaborada e apresentada a descrição e discussão dos resultados encontrados no estudo.

Os objetivos dos estudos que compuseram esta revisão bem como seus achados foram minuciosamente explorados para possibilitar a análise das abordagens das produções científicas referente à atuação do enfermeiro nos cuidados em unidades de terapia intensiva neonatal.

\section{Resultados}

Foram encontradas 10.100 resultados de busca. Após as etapas de aplicação dos filtros (Ano; Língua; Tipo de estudo) com base nos critérios de inclusão e exclusão, leitura do título, do resumo e da leitura de modo pormenorizado dos artigos completos, foram selecionados 11 artigos que possuíam relação direta com o tema, objeto e com a questão da pesquisa. Para evidenciar esse processo foi utilizado o fluxograma PRISMA apresentado a seguir:

Fluxograma 1: Processo de eleição dos estudos segundo o método PRISMA.

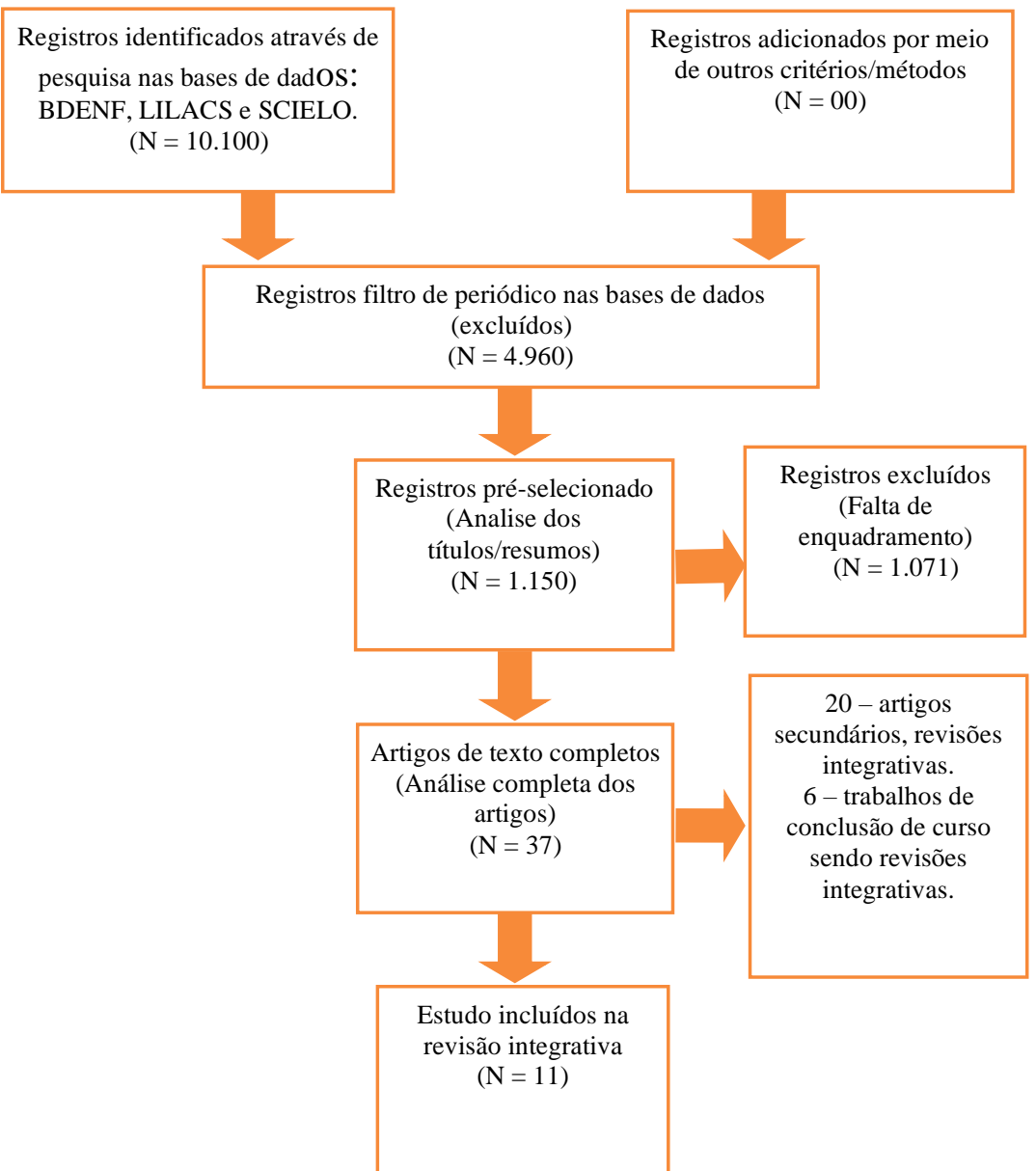

Fonte: Autores. 
A partir da análise dos artigos expostos no Fluxograma 1 e da identificação amostral de 11 (onze) estudos selecionadas, estes foram agrupados e postos nos quadros-síntese, onde foi utilizado o instrumento adaptado de Ursi \& Galvão (2005). Selecionou-se os intens: Título do artigo; Títilo do periódico; Autores; Ano de publicação, para o Quadro-síntese 1 e Metodologia; Objetivo; Resultados; Nível de evidência, para o Quadro-síntese 2:

Quadro-síntese 1: Caracterização dos estudos.

\begin{tabular}{|c|c|c|c|c|}
\hline $\mathbf{N}^{\mathbf{o}}$ & Título do artigo & Título do periódico & Autores & $\begin{array}{c}\text { Ano de } \\
\text { publicação }\end{array}$ \\
\hline 1 & $\begin{array}{l}\text { Manejo clínico da amamentação: atuação do } \\
\text { enfermeiro na Unidade de terapia intensiva neonatal }\end{array}$ & Rev Enferm UFSM & Baptista, S. S. et al & 2015 \\
\hline 2 & $\begin{array}{l}\text { O prematuro em unidade de terapia intensiva neonatal: } \\
\text { a assistência do enfermeiro }\end{array}$ & Rev enferm UFPE & $\begin{array}{l}\text { Ribeiro JF, Silva LLC da, Santos IL dos } \\
\text { et al. }\end{array}$ & 2016 \\
\hline 3 & $\begin{array}{l}\text { Escala de avaliação da dor: percepção dos enfermeiros } \\
\text { em uma unidade de terapia intensiva neonatal }\end{array}$ & Rev Enferm UFSM & Monfrim M. X. et al. & 2015 \\
\hline 4 & $\begin{array}{l}\text { Atuação do enfermeiro no cuidado ao recém-nascido: } \\
\text { proposta de um novo processo de trabalho }\end{array}$ & $\begin{array}{l}\text { Rev Enferm Cent. O. } \\
\text { Min. RECOM }\end{array}$ & $\begin{array}{c}\text { Dias MS, Ribeiro SNS, Walt CMRF, et } \\
\text { al }\end{array}$ & 2016 \\
\hline 5 & 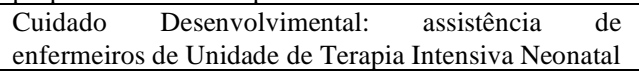 & Reben & Marski BSL, et al. & 2018 \\
\hline 6 & $\begin{array}{l}\text { Representações do cuidado de Enfermagem às mães } \\
\text { para a manutenção da lactação na Unidade de Terapia } \\
\text { Intensiva Neonatal }\end{array}$ & $\begin{array}{l}\text { J. res.: fundam. care. } \\
\text { Online }\end{array}$ & $\begin{array}{l}\text { Cherubim DO, Rodrigues AP, Paula CC; } \\
\text { et al. }\end{array}$ & 2018 \\
\hline 7 & $\begin{array}{l}\text { Diagnósticos e cuidados de enfermagem ao recém- } \\
\text { nascido prematuro em Unidade de Terapia Intensiva } \\
\text { Neonatal }\end{array}$ & $\begin{array}{l}\text { Revista Eletrônica } \\
\text { Acervo Saúde }\end{array}$ & Batista M D C, Monteiro, C J et al. & 2019 \\
\hline 8 & $\begin{array}{l}\text { Equipe de enfermagem e promoção do cuidado } \\
\text { humanizado em unidade neonatal }\end{array}$ & Rev Rene & Ferreira JHP, Amaral JJF, Lopes MMCO & 2016 \\
\hline 9 & $\begin{array}{l}\text { Cuidando do neonato que está morrendo e sua família: } \\
\text { vivências do enfermeiro de terapia intensiva neonatal }\end{array}$ & Rev Esc Enferm USP & Almeida AF, Moraes SM, Cunha RLM & 2016 \\
\hline 10 & $\begin{array}{l}\text { A ação intencional da equipe de enfermagem ao } \\
\text { cuidar do RN na UTI neonatal }\end{array}$ & Cienc. Cuid Saude & Neto SAJ, Rodrigues DRMB & 2015 \\
\hline 11 & $\begin{array}{l}\text { Assistência humanizada na terapia intensiva Neonatal: } \\
\text { ações e limitações do enfermeiro }\end{array}$ & Saúde em revista & Maria Cristina Pauli da Rocha et al. & 2015 \\
\hline
\end{tabular}

Fonte: Autores.

Quadro-síntese 2: Contribuições dos estudos.

\begin{tabular}{|c|c|c|c|c|}
\hline $\mathbf{N}^{\mathbf{o}}$ & Metodologia & Objetivo & Resultados & $\begin{array}{c}\text { Nível de } \\
\text { evidência }\end{array}$ \\
\hline 1 & $\begin{array}{l}\text { Estudo descritivo, } \\
\text { exploratório, com } \\
\text { abordagem qualitativa, } \\
\text { mediante entrevista } \\
\text { semiestruturada }\end{array}$ & $\begin{array}{l}\text { Compreender o manejo clínico } \\
\text { da amamentação realizado pelos } \\
\text { enfermeiros na Unidade de } \\
\text { Terapia Intensiva Neonatal do } \\
\text { Hospital Universitário Antônio } \\
\text { Pedro da Universidade Federal } \\
\text { Fluminense. }\end{array}$ & $\begin{array}{l}\text { Os resultados pautaram-se em } 2 \text { características: estratégias de } \\
\text { orientação dos enfermeiros na conduta clínica da amamentação } \\
\text { na UTI Neonatal; e Rede de promoção e apoio à nutriz na alta } \\
\text { hospitalar: um caminho para o sucesso da amamentação. Estas } \\
\text { deram enfoque às orientações como estratégia para o incentivo } \\
\text { e apoio à amamentação. O enfermeiro qualificado com a prática } \\
\text { do manejo clínico da amamentação colabora para o apoio ao } \\
\text { aleitamento materno e o enfrentamento do desmame precoce. }\end{array}$ & 4 \\
\hline 2 & $\begin{array}{l}\text { Estudo descritivo, com } \\
\text { abordagem qualitativa, } \\
\text { entrevistando-se } 11 \\
\quad \text { enfermeiros } \\
\text { assistencialistas de uma } \\
\text { Unidade de Terapia } \\
\text { Intensiva Neonatal. }\end{array}$ & $\begin{array}{l}\text { Analisar a assistência oferecida } \\
\text { pelo enfermeiro ao neonato em } \\
\text { uma Unidade de Terapia } \\
\text { Intensiva Neonatal. }\end{array}$ & $\begin{array}{l}\text { Demonstrou-se que o enfermeiro que atua na Unidade de } \\
\text { Terapia Intensiva Neonatal, carece de conhecimentos técnicos e } \\
\text { científicos acerca de suas atribuições específicas e privativas, } \\
\text { além de ser capacitado e qualificado para ofetarr assistência de } \\
\text { qualidade ao neonato e família. Necessidade de novas } \\
\text { estratégias na implementação das políticas de educação e } \\
\text { saúde, observando-se a necessidade de educação permanente no } \\
\text { traabalho. }\end{array}$ & 4 \\
\hline 3 & $\begin{array}{l}\text { Pesquisa qualitativa, } \\
\text { exploratória e descritiva, } \\
\text { realizada em uma } \\
\text { Unidade de Terapia } \\
\text { Intensiva Pediátrica e } \\
\text { Neonatal. }\end{array}$ & $\begin{array}{l}\text { Conhecer a percepção de } \\
\text { enfermeiros com relação à } \\
\text { utilização de um instrumento } \\
\text { para avaliação da dor em } \\
\text { neonatos prematuros }\end{array}$ & $\begin{array}{l}\text { Foram elencadas as categorias a seguir: Escala para avaliação } \\
\text { da dor nos RNs prematuros: percepção dos enfermeiros e } \\
\text { Escala de dor e o prematuro: sua implementação. Os } \\
\text { enfermeiros não conhecem as novas tecnologias usadas para } \\
\text { mensurar a dor; ainda sim, todos demostraram estar } \\
\text { comprometidos com uma assistência humanizada, visto que } \\
\text { relataram interesse pela inserção do instrumento de avaliação. }\end{array}$ & 4 \\
\hline 4 & $\begin{array}{l}\text { Estudo transversal e } \\
\text { descritivo do qual } \\
\text { participaram } 53 \\
\text { enfermeiros que atuam na } \\
\text { assistência ao recém- } \\
\text { nascido na Unidade de } \\
\end{array}$ & $\begin{array}{l}\text { Investigar a atuação } \text { do } \\
\text { enfermeiro no cuidado ao } \\
\text { recém-nascido nas unidades } \\
\text { neonatais diante desse novo } \\
\text { modelo de equipe }\end{array}$ & $\begin{array}{l}\text { Um quantitativo é especialista em neonatologia }(18,87 \%) \text {. } \\
\text { Destacaram-se a disposição dos profissionais em participar de } \\
\text { procedimentos como inserção de Cateter Central de Inserção } \\
\text { Periférica }(31,88 \%) \text { e a disposição nas discussões de casos com } \\
\text { a equipe multiprofissional }(15,94 \%) \text {.. Um bom quantitativo de } \\
\text { profissionais }(92,4 \%) \text { acredita que esse modelo pode vir a }\end{array}$ & 4 \\
\hline
\end{tabular}




\begin{tabular}{|c|c|c|c|c|}
\hline & $\begin{array}{l}\text { Terapia Intensiva e na } \\
\text { Unidade de Cuidado } \\
\text { Intermediário Neonatal. }\end{array}$ & & $\begin{array}{l}\text { melhorar a qualidade da assistência. A literatura produzida é } \\
\text { pouca sobre esse modelo assistencial de enfermagem no Brasil. } \\
\text { Este estudo registrou a atuação do profissional enfermeiro } \\
\text { nesse novo arranjo de equipe. }\end{array}$ & \\
\hline 5 & $\begin{array}{l}\text { Estudo qualitativo coleta } \\
\text { de dados pesquisa } \\
\text { documental em } \\
\text { prontuários e entrevista } \\
\text { semiestruturada e a } \\
\text { Análise de Conteúdo de } \\
\text { Bardin, como método de } \\
\quad \text { análise. }\end{array}$ & $\begin{array}{l}\text { Analisar o Cuidado Desenvolvi } \\
\text { mental na assistência de } \\
\text { enfermeiros ao Recém-Nascido } \\
\text { crítico, em Unidade de } \\
\text { Terapia Intensiva Neonatal. }\end{array}$ & $\begin{array}{l}\text { Os enfermeiros possuem conhecimento sobre do Cuidado } \\
\text { Desenvolvimental, ainda sim, há discordâncias com o fazer } \\
\text { destes. A análise apresenta-se em torno de duas categorias } \\
\text { temáticas: "Cuidado Desenvolvimental na atuação do } \\
\text { enfermeiro" e "Enfermeiro, família e Cuidado } \\
\text { Desenvolvimental". É importante trazer reflexões sobre a } \\
\text { assistência do enfermeiro quanto ao Cuidado } \\
\text { Desenvolvimental, e estimular a sensibilidade e percepção em } \\
\text { relação ao executado e registrado. }\end{array}$ & 4 \\
\hline 6 & $\begin{array}{l}\text { Estudo descritivo de } \\
\text { abordagem qualitativa }\end{array}$ & $\begin{array}{l}\text { Descrever o cuidado de } \\
\text { Enfermagem, desenvolvido } \\
\text { pelos profissionais no cotidiano } \\
\text { assistencial da Unidade de } \\
\text { Terapia Intensiva } \\
\text { Neonatal (UTIN), para a } \\
\text { manutenção da lactação. }\end{array}$ & $\begin{array}{l}\text { Estudo composto por } 10 \text { profissionais de Enfermagem, no } \\
\text { período de fevereiro de } 2013 \text {, e analisados por meio de } \\
\text { categorias temáticas. A ordenha mamária, a escuta atenta e de } \\
\text { zelo que fazem parte dos cuidados de Enfermagem para a } \\
\text { manutenção da lactação. As experiências dos profissionais de } \\
\text { Enfermagem foram apontadas como influenciadorasna } \\
\text { assistência e promoção da oferta do leite materno ao RN } \\
\text { prematuro. O cuidar na manutenção da lactaçãonão contempla } \\
\text { apenas o RN internado, sendo importante também a inclusão da } \\
\text { mãe e de seus familiares, através de grupos de apoio entre } \\
\text { mães, família e profissionais para o sucesso do aleitamento } \\
\text { materno. }\end{array}$ & 4 \\
\hline 7 & $\begin{array}{l}\text { Trata-se de Uma pesquisa } \\
\text { qualitativa, descritiva, por } \\
\text { meio de entrevista } \\
\text { semiestruturada. A } \\
\text { análise de dados se deu } \\
\text { pela técnica de Bardin. }\end{array}$ & $\begin{array}{l}\text { Descrever os rincipais } \\
\text { diagnósticos de enfermagem } \\
\text { segundo a Taxonomia NANDA- } \\
\text { I e os cuidados de enfermagem } \\
\text { ao recém-nascido prematuro em } \\
\text { uma Unidade de Terapia } \\
\text { Intensiva. }\end{array}$ & $\begin{array}{l}\text { A amostra demonstrou que a maioria dos entrevistados era do } \\
\text { sexo feminino, com média de } 11 \text { anos de atuação e } \\
\text { especialização em neonatologia. Emergiu-se, três categorias: } \\
\text { "Teoria de enfermagem para implementação da Sistematização } \\
\text { da Assistência de Enfermagem e do Processo de Enfermagem e } \\
\text { os protocolos estabelecidos para uma assistência de qualidade } \\
\text { ao recém-nascido pré-termo", "Principais diagnósticos de } \\
\text { enfermagem identificados no cuidado do recém-nascido pré- } \\
\text { termo", "Principais intervenções e/ou ações de enfermagem } \\
\text { utilizados como estratégias do cuidado integral ao recém- } \\
\text { nascido pré-termo". Pontuou-se que para um cuidado de } \\
\text { enfermagem de qualidade é imprescindível o uso da } \\
\text { Sistematização da Assistência de Enfermagem e do Processo de } \\
\text { Enfermagem. Os profissionais devem utilizar os protocolos } \\
\text { para garantir uma assistência qualificada e sistemática. }\end{array}$ & 4 \\
\hline 8 & $\begin{array}{l}\text { Pesquisa qualitativa, } \\
\text { Aplicou-se entrevista } \\
\text { semiestruturada com } \\
\text { análise de conteúdo }\end{array}$ & $\begin{array}{l}\text { Compreender conhecimentos e } \\
\text { ações da equipe de enfermagem } \\
\text { acerca do cuidado humanizado } \\
\text { em Centro de Terapia Intensiva } \\
\text { Neonatal }\end{array}$ & $\begin{array}{l}\text { Emergiram três categorias temáticas: Atenção humanizada ao } \\
\text { RN; Acolhimento à família do RN e Promoção da ambiência } \\
\text { neonatal. O manejo da equipe de Enfermagem para com RN, } \\
\text { mostrou conhecimento técnico-científico, habilidades e gestos } \\
\text { humanizados que propiciaram a recuperação do RN, } \\
\text { diminuiram os fatores estressantes no ambiente neonatal, além } \\
\text { de gerar acolhimento aos familiares e a intituição do vínculo } \\
\text { durante o processo de cuidar. Adquirir conhecimentos sobre o } \\
\text { cuidado humanizado, dispôs maior comprometimento, } \\
\text { sensibilidade, zelo no cuidar destacando, sobretudo, promoção } \\
\text { de conforto, segurança do RN, acolhimento à família e atenção } \\
\text { na ambiência. }\end{array}$ & 4 \\
\hline 9 & $\begin{array}{l}\text { Trata-se de um estudo } \\
\text { descritivo exploratório, de } \\
\text { abordagem qualitativa }\end{array}$ & $\begin{array}{l}\text { Compreender as experiências } \\
\text { vivenciadas pelos enfermeiros } \\
\text { ao cuidar de neonatos que estão } \\
\text { morrendo e sua família na } \\
\text { UTIN e resgatar as percepções } \\
\text { destes profissionais em relação } \\
\text { à sua atuação diante do processo } \\
\text { de morte e luto vivenciado. }\end{array}$ & $\begin{array}{l}\text { A dificuldade encada pelos enfermeiros aoprestar assistência ao } \\
\text { neonato que está evoluindo a óbito e sua família, mostra-se } \\
\text { muito eloquente nos discursos. Os envolvimento emocional é } \\
\text { inevitável, pois esses profissionais acompanham esse RN } \\
\text { constantemente em seu turno de trabalho, desde a admissão até } \\
\text { o momento de seu óbito ou alta. }\end{array}$ & 4 \\
\hline 10 & $\begin{array}{l}\text { Estudo de natureza } \\
\text { qualitativa, com } \\
\text { abordagem } \\
\text { fenomenológica e } \\
\text { referencial teórico- } \\
\text { metodológico de Alfred } \\
\quad \text { Schutz }\end{array}$ & $\begin{array}{l}\text { Apreender o que a Equipe de } \\
\text { Enfermagem tem em vista ao } \\
\text { cuidar do Recém-Nascido na } \\
\text { Unidade de Terapia Intensiva } \\
\text { Neonatal. }\end{array}$ & $\begin{array}{l}\text { Os discurssos foram adquiridos através da entrevista } \\
\text { fenomenológica, onde a ação intencional foi aplicada mediante } \\
\text { as seguintes categorias: buscar o melhor cuidado e a melhora } \\
\text { do RN; reconhecer a perspectiva humana do cuidado na UTI } \\
\text { Neonatal; e Agir com responsabilidade. A ação dos cuidadores } \\
\text { de Enfermagem ao exercerem sua assistência ao RN mostrou-se } \\
\text { como uma atuação de responsabilidade, preocupação, atenção, } \\
\text { carinho e sensibilidade, causando um senso crítico e reflexivo } \\
\text { acerca dos aspectos éticos e humanos inerentes às suas ações. } \\
\text { Os resultados apontam: repensar novas maneiras de cuidar, } \\
\text { utilizando a arte e a criatividade e humanização das } \\
\text { tecnologias, incluindo a ética como temática importante na } \\
\text { produção e no desenvolvimento de novos saberes. }\end{array}$ & 4 \\
\hline 11 & $\begin{array}{c}\text { Estudo qualitativo } \\
\text { utilizado como referencial }\end{array}$ & $\begin{array}{l}\text { Compreender a experiência do } \\
\text { enfermeiro de UTI Neonatal em }\end{array}$ & $\begin{array}{l}\text { Os participantes do estudo foram compostos por sete } \\
\text { enfermeiras que atuavam na Unidade de Terapia Intensiva }\end{array}$ & 4 \\
\hline
\end{tabular}




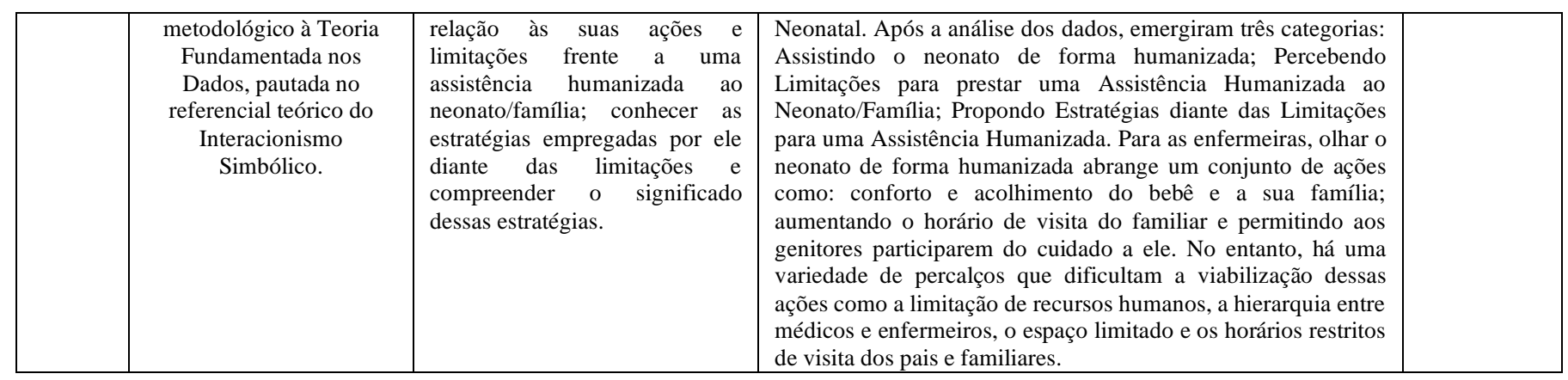

Fonte: Autores.

Os resultados encontrados abordaram as seguintes categorias temáticas: $\mathbf{O}$ papel do enfermeiro na assistência da UTIN e A sistematização da assistência de enfermagem (SAE) aplicada em UTIN.

\subsection{Categoria Temática 1: O papel do enfermeiro na assistência da UTIN}

Neste tema, pos-se em evidência que os enfermeiros atuantes em UTIN têm graus de formação diferenciados e a estruturação do trabalho ocorre pela divisão de tarefas entre os membros dos trabalhadores de enfermagem. Este efetua o trabalho de maior proporção científica/intelectual, burocrática e de gerenciamento de trabalho assistencial, enquanto os demais membros da equipe de enfermagem são executores de tarefas rotineiras e delegadas sob a supervisão do enfermeiro (Dias et al., 2016).

Dentro de suas habilidades e competências, o enfermeiro possui a responsabilidade de cuidar cotidiana e intensamente do RN, o que inclui a parte assistencial direta, mas não somente deste, pois atuam também prestando esclarecimentos e orientações aos familiares e cuidadores acerca dos cuidados específicos com o mesmo. Assim, o enfermeiro e sua equipe são responsáveis por proporcionar o amoldamento do RN ao ambiente exterior, com uso de tecnologias e condutas que visam a estabilidade térmica, umidade, luz, estímulos sonoros e cutâneos; observação da situação clínica; monitorização dos sinais prognósticos e a evolução do tratamento desse RN; atenção às necessidades vitais para este; elaboração e implementação um plano educativo em saúde; além disso, coordenar e supervisionar a assistência prestada no setor (Ribeiro et al., 2016).

Ademais, se exercem procedimentos técnicos que competem apenas ao enfermeiro, realizados em uma UTIN, caracterizados por: inserção de sondas oro ou nasogástrica e enteral, e sondagem por via vesical; realização do Cateter Central de Inserção Periférica (PICC) - quanto o procedimento é padronizado nas instituições e o profissional é capacitado; realização de gasometria venosa ou arterial e amostra para cultura sanguínea; aspiração via oral-traqueal e do tubo endotraqueal, assim como, efetua curativos de grande complexidade e analisa escalas e índices conforme rotina do setor (Ribeiro et al., 2016).

O/a enfermeiro/enfermeira estão diretamente ligada na prevenção da infecção neonatal, por passar a maioria do tempo em cuidado e vigilância dos RN e assim, têm uma responsabilidade maior com o cuidado, de tal modo cabe a ela junto de outros profissionais contribuir para a sua prevenção. A enfermagem também desempenha o papel fundamental na avaliação e minimização da dor, de modo a intervir no curso de manifestações clínicas que interfiram no conforto. Os enfermeiros também executam estratégias fomentar a amamentação, tais como a orientação da pega correta, higiene das mamas e ordenha, as quais se incluem no processo do aleitamento materno, e são instrumentos de educação que compartilham o conhecimento às nutrizes (Baptista et al., 2015; Monfrim et al., 2015).

Observa-se ainda que a efetuação da atenção humanizada ao RN preconiza intervenções que se referem à individualidade e integralidade do cuidado, ainda que associada ao uso de tecnologias duras para a recuperação do RN, garantia de acolhimento à família, estabelecimento do vínculo e apego, dentre outras. Estudo sobre humanização do cuidado mostra resultados semelhantes, além de ressaltar a importância de se estabelecer comunicação e interação de forma efetiva e afetiva, durante todo processo assistencial (Ferreira, Amaral \& Lopes, 2017). 


\subsection{Categoria Temática 2: A Sistematização da Assistência de Enfermagem (SAE) Aplicada em UTIN}

A utilização da SAE proporciona cuidados individualizados, assim como, norteia a ação decisória e a administração da equipe. Oportuniza avanços na destreza assistencial, o que impulsiona sua adoção nas instituições que dispõe à assistência à saúde. O diagnóstico de enfermagem (DE) é uma etapa essencial do processo de enfermagem PE, uma ferramenta para a aplicabilidade da SAE que contribui e orienta para um cuidado que visa um alto padrão de qualidade e organizado. Nessa etapa, ocorre um julgamento clínico sobre uma condição de saúde, que tem efeito direto no tratamento, orientando de forma significativa a tomada de decisão e intervenções de enfermagem (Silva et al., 2019).

A SAE na UTIN se configura como uma ação de suma importância, pois não se limita apenas a manter o neonato com vida, mais também a monitorar e acompanhar sua evolução e as suas particularidades (RIBEIRO et al., 2016). Os Diagnósticos de Enfermagem (DE), segunda etapa do PE - Taxonomia II da NANDA Internacional (NANDA-I) - é uma linguagem padronizada que viabiliza a comunicação sobre os fenômenos da prática de enfermagem. Desta forma, verificou-se que os cuidados ao neonato em uma UTIN demanda grande responsabilidade, onde faz-se, sempre que há necessidade, intervenção da equipe de enfermagem de forma resolutiva, com intuito de contornar possíveis situações de agravamentos vivenciadas (Medeiros et al., 2016).

Os principais diagnósticos de enfermagem evidenciados estão relacionados aos riscos, tais como: risco de infecção, risco de hipotermia e risco de integridade da pele prejudicada. Os diagnósticos reais mais relevantes foram os relacionados aos múltiplos sistemas orgânicos do RN.

\subsection{Principais diagnósticos de enfermagem evidenciados a partir da NANDA-I 2018-2020}

\subsubsection{Diagnósticos de enfermagem relacionado à pele, mucosa e anexos:}

Hiperbilirrubinemia neonatal, relacionada à idade $\leq 7$ dias; relacionada à atraso na eliminação do mecônio; relacionada ao perfil sanguíneo incompatível com o da mãe, caracterizada por pele, esclerótica e mucosas amareladas e perfil sanguíneo anormal.

Risco de hiperbilirrubinemia neonatal, relacionado à dificuldade de realizar a transição entre à vida intrauterina há extrauterina; relacionado a idade neonatal $\leq 7$ dias; relacionado a prematuridade e relacionado a imaturidade do fígado.

Hipotermia, relacionado à banho antecipado do RN, caracterizada por temperatura central de $36-36,5^{\circ} \mathrm{C}$, pele fria ao toque e cianose de extremidades.

Risco de hipotermia, relacionado a instabilidade hipotalâmica; relacionado a banho precoce, relacionado a fatores ambientais; relacionado a deficiência de gordura marrom e relacionado a atividade muscular reduzida.

Integridade da pele prejudicada, relacionada à alteração na pigmentação, caracterizada por petéquias; relacionada à reação alérgica, caracterizada por pequenas lesões avermelhadas.

Risco de integridade da pele prejudicada, relacionado a circulação ineficiente, hipotermia e prematuridade.

Mucosa oral prejudicada, relacionada a alteração da forma anatômica (lábio Leporino), caracterizada por impossibilidade de sucção e; relacionada ao uso de dispositivo ventilatório, caracterizado por lesões, abrasões e hemorragias gengivais.

\subsubsection{Diagnósticos de enfermagem relacionado trato gastrointestinal:}

Amamentação interrompida, relacionada à doença do lactente, hospitalização e separação da mãe, caracterizada por alimentação materna não exclusiva; relacionada a incapacidade de eliminação do mecônio, caracterizada por amamentação ineficaz; relacionada a defeito orofaríngeo, caracterizado pela suç̧ão ineficaz. 
Risco de amamentação ineficaz, relacionado a prematuridade; relacionado a internação em UTI; relacionado a deficiência do conhecimento da técnica da pega correta.

Leite materno insuficiente, relacionado a deficiência de produção, caracterizado por quantidade produzida menor que a necessidade do recém-nascido.

Risco de motilidade gastrointestinal disfuncional, relacionado a prematuridade.

\subsubsection{Diagnósticos de enfermagem relacionados ao sistema neurológico:}

Comportamento desorganizado do lactante, relacionado à doença neurológica congênita, caracterizado por reação prejudicada aos estímulos sensoriais.

Desenvolvimento motor e mental eficaz, relacionado a capacidade de agilidade e inteligência, evidenciado pela facilidade de sucção e esperteza.

Função neurológica alterada, relacionada à microcefalia, caracterizada por déficit cognitivo; relacionado a macrocefalia, caracterizado por hidrocefalia congênita; relacionado a macrocefalia, caracterizado por hematomas crônicos e/ou sangramento intracraniano; e relacionado a síndrome de Huler, caracterizado por macrocefalia.

\subsubsection{Diagnósticos de enfermagem relacionados ao sistema respiratório:}

Desobstrução ineficaz das vias aéreas, relacionada a muco excessivo e secreções retidas, caracterizada por cianose, dispneia e batimentos de asas nasais; relacionado a excesso de secreção, caracterizado por ruídos adventícios e murmúrios vesiculares diminuídos.

Ventilação espontânea prejudicada, relacionada a instabilidade pulmonar, caracterizada por auxílio da musculatura acessória; relacionada a instabilidade pulmonar, caracterizada por $\mathrm{SpO} 2<80 \%$, cianose central, agitação, taquicardia e taquidispneia.

Instabilidade pulmonar, relacionada a prematuridade, caracterizado por retrações costais, gemido expiratório, ruídos adventícios (roncos, sibilos e estertores).

\subsubsection{Diagnósticos de enfermagem relacionados ao sistema cardiovascular:}

Função cardiovascular prejudicada, relacionada ao retardo da transferência de circulação fetal para neonatal, caracterizado por acrocianose, taquipneia e agitação.

Débito cardíaco diminuído, relacionado a função cardíaca alterada, caracterizado por bradicardia e perfusão tissular periférica > que 5 segundos; e relacionado ao ritmo cardíaco alterado, caracterizado por alterações eletrocardiográficas.

\subsubsection{Diagnósticos de enfermagem relacionados ao sistema renal, endócrino e metabólico:}

Risco de hipoglicemia, relacionado a amamentação ineficaz; relacionado a deficiência de leite materno.

Hipocalcemia, relacionada ao grau de maturação da paratireoide neonatal, caracterizada por diminuição da excitabilidade e contração muscular.

Desidratação relacionada a perda ativa de volume de líquidos e instabilidade renal, caracterizada pela diminuição do débito urinário.

\subsubsection{Diagnósticos de enfermagem relacionados ao risco de morte:}

Risco de morte súbita do lactente, relacionado a deficiência de cuidados no pré-natal; relacionado ao baixo peso ao nascer; relacionado a hipotermia; e relacionado a cuidados ineficientes no período neonatal. 


\section{Discussão}

A categoria temática 1 evidencia que o desafio dos profissionais da enfermagem atuantes da UTI Neonatal contorna as ações que visam garantir características adequadas para vincular um cuidado integral da saúde do neonato conjuntamente a atenção materna e familiar, especialmente a manutenção do aleitamento materno. $\mathrm{O}$ enfermeiro busca desenvolver uma atenção eficaz e acolhedora em prol da qualidade no cuidado, visando sempre a promoção e o apoio à amamentação como qualidade de vida tanto para o bebê quanto para a mãe (Baptista et al., 2015).

Os enfermeiros inserem-se também nas atividades atreladas à administração da instituição, dos recursos materiais e humanos, da logística organizacional e supervisão no cuidar (Ramos et al., 2021). Ainda destaca-se o processo de trabalho da equipe de enfermagem no desenvolvimento da $\mathrm{SAE}$ ao $\mathrm{RN}$, pois é através deste trabalho que se pode garantir a prática profissional de forma resolutiva, padronizada e humanizada (Ribeiro et al., 2016).

Destaca-se também que o enfermeiro da UTIN deve estar preparado para lidar com as instabilidades hemodinâmicas de pacientes em situações críticas, sobrevivendo a condições adversas, em um local tido como árduo e que requer aptidões profissionais para enfrentar situações difíceis e garantir o atendimento integral e resolutivo do paciente (Alencar et al., 2016).

A categoria 1 expressa ainda que os profissionais ressaltaram sua prática, mostrando-se capazes de prestar assistência individualizada e humanizada, essencial à sobrevivência e recuperação da saúde dos bebês. O cuidado oferecido ao neonato se expande além do cuidado técnico e da compreensão científica. Mediante as práticas mencionadas, identificaram-se cuidados em relação ao conforto, proteção ao bebê, com atenção às especificidades do atendimento humanizado e estendido à família. Logo, a sensibilidade da equipe de enfermagem retrata a existência do cuidar com zelo, respeito, individualizado e importante para o bem-estar e qualidade de vida (Ribeiro et al., 2016).

Para promover e apoiar a amamentação, o profissional da enfermagem, além do conhecimento em aleitamento materno e competência clínica, precisa possuir habilidade de comunicação efetiva com a nutriz, sendo necessário abordar os princípios básicos que implicam em acolher, ajudar a mulher a tomar decisões de forma empática, saber ouvir, apreender, desenvolver a confiança e prestar apoio. No caso do enfermeiro, seu papel é o de informar as mães e familiares sobre os benefícios do leite materno, fornecer suporte e cuidado à mulher nutriz e seu filho, propor intervenções para obter uma lactação efetiva e fortalecer o binômio e família (Baptista et al., 2015).

Orientar a mulher/nutriz para a grande relevância da prática do aleitamento materno constitui a possibilidade de promover a educação em saúde, com as devidas orientações acerca da posição do bebê e da pega correta. Além disso, as orientações, iniciadas ainda durante a internação da criança, podem contribuir para eliminar dúvidas e superar obstáculos impeditivos para o sucesso da prática da amamentação e, ainda, para prevenir futuros problemas mamários que possam dificultar o aleitamento exclusivo (Baptista et al., 2015).

A humanização do cuidado envolve a inserção da família durante a internação do bebê, cujos profissionais devem preparar os pais a participarem do processo de cuidar. Neste estudo, percebeu-se empatia, respeito, durante encontro entre profissionais, neonato e familiares, visto como momento oportuno que beneficia a identificação das necessidades, estabelecimento do vínculo afetivo, bem como possibilita segurança para cuidar do filho. A equipe de enfermagem acolhe os familiares com orientações necessárias, quanto às rotinas institucionais e dúvidas a respeito da doença e do tratamento, resultando aproximação desse binômio, fortalece os laços afetivos, bem como atenua efeitos negativos da internação (Alencar et al., 2016).

A categoria temática 2 destaca a utilização da SAE como uma das atividades privativas do enfermeiro que visa organizar, padronizar os cuidados ao neonato em UTIN. Nesta, os conteúdos dos artigos demonstraram que a SAE se encontra implementada nas diversas UTIN, que é aplicada e ainda que é tida como fundamental ferramenta na organização e gerenciamento das atividades de enfermagem (Ribeiro et al., 2016). 
Destaca-se que a SAE propicia avanços significativos na assistência e desenvolvimento de boas práticas de enfermagem e vem melhorando os resultados assistenciais aos neonatos, diminuindo a morbimortalidade e aumentando a sobrevivência de prematuros de baixo peso e extremo baixo peso. Porém salienta-se que a atividade em UTIN exige a incorporação de profissionais com grade experiência e capacitação. Inclusive devido ao fato de o enfermeiro dividir seu tempo entre as suas atividades de cuidados de alta complexidade e trabalho no gerenciamento e, desta forma, delega os demais cuidados neonatais rotineiros aos técnicos de enfermagem (Ferreira, Amaral \& Lopes, 2017).

Especificamente com relação a base legal da SAE, na Resolução do Conselho Federal de Enfermagem (COFEN) n ${ }^{\circ}$ 358/2009 esse processo se organiza em cinco etapas, coleta de dados, diagnóstico, planejamento, implementação e avaliação de enfermagem, onde o/a enfermeiro/a põe em prática o seu conhecimento teórico e proporcionar uma assistência melhor ao paciente (Cofen, 2009).

A taxonomia de Diagnósticos de Enfermagem (DE), segunda etapa do PE é uma linguagem padronizada que viabiliza a comunicação sobre os fenômenos da prática de enfermagem e avalia o cuidado, utilizando-se do julgamento clínico do enfermeiro para diagnosticar as respostas humanas às condições de saúde reais ou potenciais de risco (Medeiros, et al., 2016).

A assistência na UTI neonatal está centrada no corpo biológico e em fatores psicossociais, emocionais e espirituais. Assim, o sucesso ou insucesso na aplicação da SAE depende de fatores-chave como apoio, colaboração e interesse das chefias de enfermagem, além do preparo rigoroso, constante e sistemático dos enfermeiros. Entretanto, o quantitativo reduzido de profissionais e a sobrecarga de trabalho dos enfermeiros são destacadas como fatores que atrapalham a qualidade da assistência sistematizada nas UTIN (Ribeiro et al., 2016).

\section{Conclusão}

O estudo evidenciou o compromisso dos profissionais no cuidar do RN, com destaque as atitudes de zelo, segurança, respeito à individualidade e especificidades, bem como, retrata os esforços para qualificação dos cuidados aplicados, minimização dos riscos inerentes aos procedimentos de alta complexidade e garantir a ambiência do setor, buscando torná-lo menos agressivo ao neonato. Verificou-se também o avanço em relação ao acesso dos familiares à UTIN, sobretudo mãe; além de atenção especial a sua participação nos cuidados ao $\mathrm{RN}$, favorecendo a amamentação e o vínculo, preconizados pelas políticas humanização.

O estudo também apontou o protagonismo na SAE e a sua significativa contribuição à assistência nas UTIN, conforme com as etapas processuais, que são essenciais no objetivo de se garantir a organização do trabalho e o respaldo técnico-científico de toda a equipe, por meio do planejamento, organização, execução do cuidado.

A SAE ainda fornece subsídios à assistência, proporcionando resultados positivos e de base científica, sendo fundamental, pois proporciona ao enfermeiro não se limitar a garantir a sobrevida do RN, mas também atender suas necessidades específicas de cuidado ao RN e seus familiares.

As questões abordadas neste estudo fornecem baes para novas pesquisas e sinalizam para a questão de um quantitativo pequeno de debate nesta área. Pode-se destacar a relevância de elevar o número de pesquisas voltadas ao tema, objetivando ofertar subsídios para condutas ou ações futuras que visem minimizar viéses e melhorar as condutas e os cuidados da equipe de enfermagem para com o binômio mãe e filho e seus familiares.

\section{Referências}

Alencar, A. P. A. et al. (2017). Atuação do Profissional de Enfermagem na Unidade de Terapia Intensiva (UTI). Revista E-Ciência, 4(2).

Baptista, S. S. et al. (2015). Manejo clínico da amamentação: atuação do enfermeiro na Unidade de Terapia Intensiva Neonatal. Revista de Enfermagem da UFSM, 5(1), 23-31. 
Research, Society and Development, v. 10, n. 6, e1910614588, 2021

(CC BY 4.0) | ISSN 2525-3409 | DOI: http://dx.doi.org/10.33448/rsd-v10i6.14588

Coelho, A. D. S. et al. (2018). Nursing team and humanized assistance in neonatal UTI. ReonFacema, 49(69), 873-877.

Dias, M. S. et al. (2016). Atuação do enfermeiro no cuidado ao recém-nascido: proposta de um novo modelo. Revista de Enfermagem do Centro-Oeste Mineiro, 1(6), 1930-1944.

Ferreira, J. H. P. \& Amaral, J. J. F. \& Lopes, M. M. C.O. (2017). Equipe de enfermagem e promoção do cuidado humanizado em un idade neonatal. Revista da Rede de Enfermagem do Nordeste, 17(6), 741.

Gomes, D. F. et al. (2019). Papel do enfermeiro no cuidado intensivo neonatal no Brasil. Essentia (Sobral), 20(1), 9-16.

Herdman H, Kamitsuru S. Diagnósticos de enfermagem da NANDA: definições e classificação 2018-2020. Artmed; 2018

Medeiros, A. L. et al. (2016). Avaliando diagnósticos e intervenções de enfermagem no trabalho de parto e na gestação de risco. Rev Gaúcha Enferm. 37(3), 55316.

Monfrim, X. M. et al. (2015). Escala de avaliação da dor: percepção dos enfermeiros em uma Unidade de Terapia Intensiva Neonatal. Revista de Enfermagem da UFSM, 5(1), 12-22.

Mombaque, W. \& Secoli, S. R. A. (2018). Abordagem do Joanna Briggs Institute para revisões sistemáticas. Carta ao Editor.

Pereira, A. S., et al. (2018). Metodologia da pesquisa científica. UFSM. https://repositorio.ufsm.br/bitstream/handle/1/15824/Lic_Computacao_MetodologiaPesquisa-Cientifica.pdf?sequence $=1$.

Oliveira, S. R. et al. (2017). Assistência de enfermagem ao recém-nascido prematura na unidade de terapia intensiva neonatal. In: International Nursing Congress, Anais, 9-12.

Ramos, C. S. et al. (2021). Estresse ocupacional presente nas atividades da equipe de enfermagem em centro cirúrgico: revisão integrativa. Research, Society and Development, 10(3), e13310413872. http://dx.doi.org/10.33448/rsd-v10i4.13872

Resolução no 358, de 15 de outubro de 2009. Sistematização da Assistência de Enfermagem e implementação do Processo de Enfermagem em ambientes, públicos ou privados, em que ocorre o cuidado profissional de Enfermagem. Conselho federal de Enfermagem - COFEN. Brasília.

Ribeiro, J. et al. (2016). O Prematuro Em Unidade De Terapia Intensiva Neonatal: a Assistência Do Enfermeiro. Journal of Nursing UFPE/Revista de Enfermagem UFPE, 10(10).

Ribeiro, R. F. et al. (2015). Humanização na Unidade de Terapia Intensiva. Enfermagem em Unidade de Terapia Intensiva.

Silva, D. V. A. et al. (2019). Diagnósticos de enfermagem em programa domiciliar: mapeamento cruzado e Taxonomia da NANDA-I. Rev. Bras. Enferm, 72(3), 584-591.

Silva, V. M. da, \& Tonon, T. C. A. (2020). Atuação do enfermeiro no processo da amamentação. Research, Society and Development, 9(10), e7819109158. https://doi.org/10.33448/rsd-v9i10.9158

Soares, C. B. et al. (2014). Revisão Integrativa versus Revisão Sistemática. Reme: Revista Mineira de Enfermagem, 12(4), 758-764.

Teixeira, E. et al. (2014). RIL métodos de revisão. Revista de Enfermagem da UFPI, 2(5), 3.

Ursi, E. S. (2005). Prevenção de lesões de pele no perioperatório: revisão integrativa da literatura. [dissertation]. Ribeirão Preto: Universidade de São Paulo, Escola de Enfermagem de Ribeirão Preto. 